\title{
Stacking Geometries of Early Protoporphyrin IX Aggregates Revealed by Gas-Phase Infrared Spectroscopy
}

\author{
Jongcheol Seo, ${ }^{\dagger}$ Jinhyeong Jang ${ }^{\ddagger}$ Stephan Warnke, ${ }^{\dagger}$ Sandy Gewinner, ${ }^{\dagger}$ Wieland Schöllkopf, ${ }^{\dagger}$ \\ and Gert von Helden*, \\ ${ }^{\dagger}$ Fritz-Haber-Institut der Max-Planck-Gesellschaft, Faradayweg 4-6, 14195 Berlin, Germany \\ ${ }^{\ddagger}$ Department of Chemistry, School of Natural Science, Ulsan National Institute of Science and Technology, Ulsan 44919, South \\ Korea
}

\section{Supporting Information}

ABSTRACT: Amphiphilic porphyrins are of great interest in the field of supramolecular chemistry because they can be fabricated into highly ordered architectures that are stabilized by $\pi-\pi$ stacking of porphine rings as well as by non-covalent interactions between their hydrophilic substituents. Protoporphyrin IX (PPIX) has two flexible propionic acid tails and is one of the most common amphiphilic porphyrins. However, unlike other PPIX analogues, PPIX does not form stable extended nanostructures, and the reason for this is still not understood. Here, we employ ion mobility mass spectrometry in combination with infrared multiple photon dissociation spectroscopy to investigate early aggregates of PPIX. The ion mobility results show

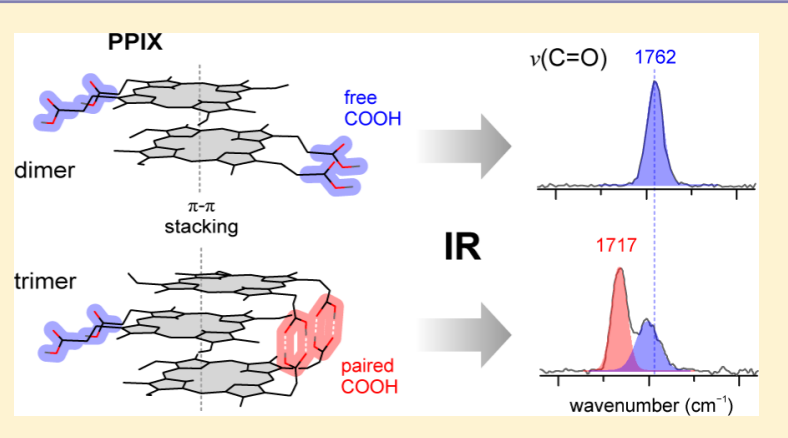
that growth occurs via single-stranded face-to-face stacking of PPIX. From the infrared spectroscopy on well-defined aggregates, it can be concluded that pairing of the carboxylic acid groups of the tails is a stabilizing element and that such a pairing occurs across a third residue from residue $n$ to residue $n+2$. The tetramer appears to be especially stable, because all of its propionic acid tails are optimally paired and no free tails to promote further growth are present, which possibly prevents PPIX from forming larger, well-ordered assemblies.

\section{INTRODUCTION}

Their flat heterocyclic structure with its delocalized $\pi$-electron system gives porphyrins unique electronic properties and makes them valuable templates for building functionalized supramolecular assemblies. Examples include porphyrin-fullerene complexes, ${ }^{1-5}$ one-dimensional fibrillar or tubular arrays of porphyrin derivatives, ${ }^{6-13}$ discotic liquid crystals, ${ }^{14-18}$ metalorganic frameworks, ${ }^{19,20}$ and several others. ${ }^{21-23}$ In such supramolecular assemblies, the well-defined redox, photochemical, and spectroscopic properties of porphyrins can be tuned, and various intermolecular interactions can drive porphyrins into specific supramolecular structures. These properties make porphyrin assemblies valuable in diverse applications in the fields of, for example, photovoltaics and photocatalysis. $^{24-28}$

Much of the stabilization in those assemblies stems from $\pi-\pi$ stacking interaction. ${ }^{29}$ However, the presence of polar, hydrophilic substituents offers the possibility of additional noncovalent interactions, and such amphiphilic porphyrins are of great interest in the design of functionalized, highly ordered supramolecular assemblies. Therefore, characterizing the noncovalent interactions of the hydrophilic substituents and correlating them to the structures of the final assemblies are of fundamental importance for the design of porphyrin nanostructures.
Protoporphyrin IX (PPIX), shown in Figure 1a, has two flexible propionic acid groups on the edge of the rigid porphine ring and is one of the most common natural amphiphilic porphyrins. Since the propionic acid tails can be easily modified, various PPIX derivatives, such as bis-glycosamide-, amine-, ${ }^{7}$ and amide-derived PPIX, ${ }^{10,11,13,30}$ have been used to build highly ordered nanostructures that include elongated 1-D micellar fibers, rods and tubes, ${ }^{6,7,11-13}$ or 3-D spherical vesicles and micelles. ${ }^{30}$ In those nanostructures, the self-assembled structures and their stabilities highly depend on the type of the hydrophilic tails and their intermolecular interactions. For example, in PPIX assemblies containing amide-derived tails, stabilization occurs via cooperative hydrogen bonding between $-\mathrm{C}=\mathrm{O}$ and $-\mathrm{N}-\mathrm{H}$ groups, ${ }^{6,10}$ and in those containing aminederived tails, amine-ammonium interactions are suggested to stabilize their supramolecular structures (Figure 1c).

Unlike these PPIX derivatives, however, PPIX itself does not form stable highly ordered structures. ${ }^{31}$ While PPIX does form higher aggregates in the $\mathrm{pH}$ range 3-7, microscopy studies rather suggest the formation of globular, vesicular structures and moreover, these aggregates are not particularly stable and can easily collapse into amorphous substances. ${ }^{31}$ Using UV/vis spectroscopy and monitoring the splitting of the Soret band,

Received: August 19, 2016

Published: November 28, 2016 


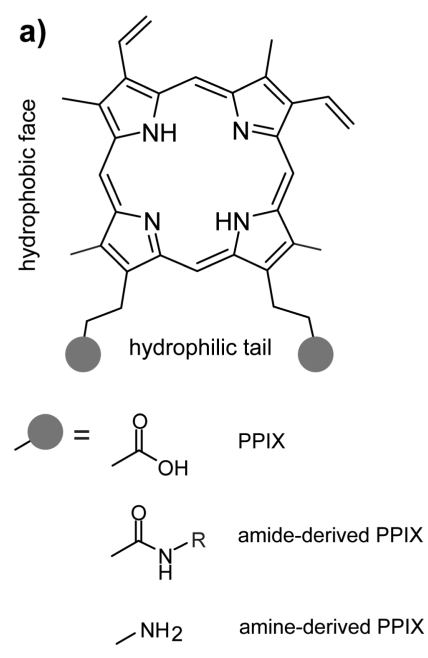

b)

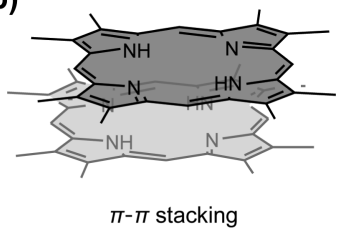

c)

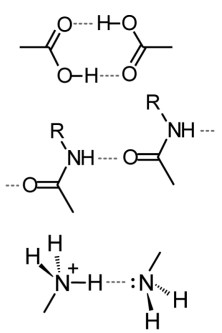

hydrogen bondings

Figure 1. (a) Chemical structures of protoporphyrin IX (PPIX) and its derivatives. $(b, c)$ Possible non-covalent interactions in PPIX aggregates; $\pi-\pi$ stacking (b) and various hydrogen bonds which can be formed between hydrophilic tails (c).

face-to-face $\pi-\pi$ stacking of the porphine rings and the formation of so-called $\mathrm{H}$-aggregates was suggested. ${ }^{31}$ Based on the slip angle between porphyrin planes obtained by the resonance light scattering, it is suggested that the propionic acid tails of one PPIX unit forms specific hydrogen bonded pairs with the tails of other PPIX units in an alternate fashion. ${ }^{31}$ This would allow to generate stable higher aggregates but the direct experimental evidence for the role of propionic acid tails is still missing.

Infrared (IR) spectroscopy is well suited to investigate the interactions of such hydrophilic tail groups, as the band positions of $\mathrm{C}=\mathrm{O}$ and $\mathrm{C}-\mathrm{OH}$ stretching vibrations as well as bending vibrations of $\mathrm{C}-\mathrm{O}-\mathrm{H}$ groups can be used to deduce information about their local interactions. However, in the condensed phase, aggregates usually coexist in a wide distribution of aggregation states and only ensemble-averaged results can be obtained.

The combination of ion mobility mass spectrometry (IMMS) and IR multiple photon dissociation (IRMPD) ${ }^{32}$ can address the above-mentioned limitation, and recent studies have shown that this combination is useful for cluster- and isomer-specific studies. For example, different protomers of aminobenzoic acids and their derivatives could be separated by IM-MS, and their protonation sites were unambiguously identified using IRMPD spectroscopy. ${ }^{33,34}$ For peptides, different structures induced by cis/trans-isomerization of a proline could be separated and determined. ${ }^{35,36}$ Further, IMMS coupled to IRMPD was used for the structural characterization of peptide aggregates ${ }^{37}$ as well as of proteins. ${ }^{38}$

IM-MS by itself allows to accurately measure the collision cross-section (CCS) of mass-to-charge $(\mathrm{m} / \mathrm{z})$ selected species. The evolution of the CCS as a function of the aggregate size reveals information about overall growth properties. Furthermore, IM-MS can be used as a preparative tool to separate oligomers of different size $n$ but identical $\mathrm{m} / z$ ratio (for example a singly charged dimer and a doubly charged tetramer). In combination with IRMPD spectroscopy, this allows the observation of vibrational spectra of individual isolated oligomers with well-defined size and shape to provide critical insights into the self-assembly process.

Here, we use IM-MS combined with IRMPD spectroscopy to study the early aggregates of PPIX. The CCS values and/or the IR spectra of $\mathrm{m} / z$ and ion-mobility selected PPIX aggregates are measured for sizes ranging from the monomer to the dodecamer. The evolution of CCS values provides insight into the overall growth geometries and the IR spectra provide further information into the hydrogen-bonding patterns of the propionic acid tails. When these data are taken together, a consistent picture of the structures and the growth properties of PPIX aggregates is obtained. These results are compared and correlated with previous findings in the condensed phase, and general conclusions on the assembly of PPIX and its derivatives are drawn.

\section{MATERIALS AND METHODS}

Sample Preparation. Protoporphyrin IX (PPIX) and all solvents were purchased from Sigma-Aldrich (Taufkirchen, Germany) and used
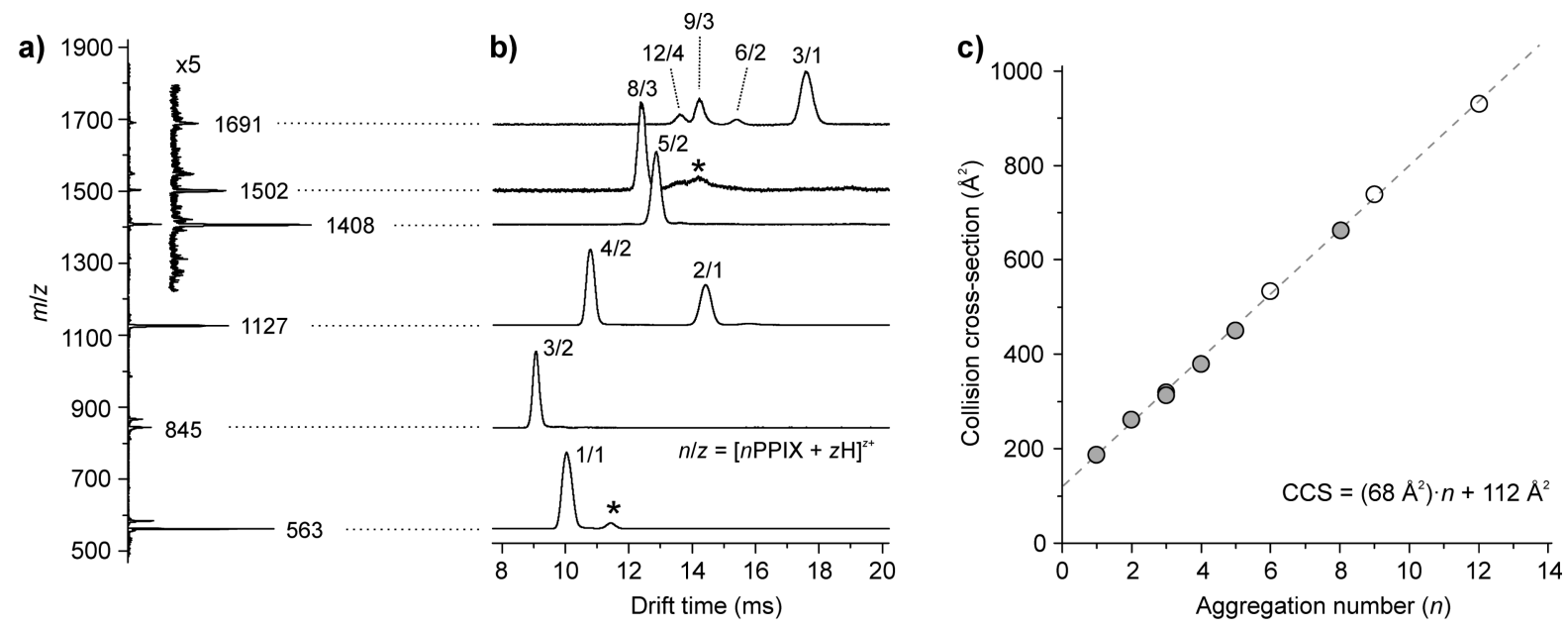

Figure 2. (a) NanoESI-MS spectrum of protoporphyrin IX, (b) arrival time distribution (ATD) of each $m / z$ species, and (c) the determined collision cross-section (CCS) values as a function of aggregation number. The $n / z$ label above each ATD peak represents $[n \text { PPIX }+z \mathrm{H}]^{z+}$, where $n$ and $z$ denote the number of monomer units (aggregation number) and charge of the complex, respectively. ATD peaks with an asterisk (*) are artifacts. The gray dashed line denotes the result of a linear regression between CCS and aggregation number. Filled circles denote the aggregates that were further investigated with gas-phase IR spectroscopy. 
without further purification. A sample solution $(100 \mu \mathrm{M})$ is prepared by dissolving PPIX in chloroform/methanol $(1 / 1, \mathrm{v} / \mathrm{v})$. An aliquot $(5-10 \mu \mathrm{L})$ of this sample solution is then loaded into a Pd/Pt-coated borosilicate nanoelectrospray ionization (nanoESI) source.

Ion Mobility Mass Spectrometry. The sample solution is electrosprayed with a spray voltage of $0.6-0.7 \mathrm{kV}$ and the generated ions are transferred into a custom-built drift tube ion mobility mass spectrometer, which is described in detail elsewhere. ${ }^{39-41}$ In this instrument, the ions are initially collected in an RF ion funnel from which they are pulsed with 10 or $20 \mathrm{~Hz}$ repetition rate into a $80 \mathrm{~cm}$ long drift tube filled with helium buffer gas (4-5 mbar). Ions traverse the drift tube under the influence of a weak electric field (10-15 V $\mathrm{cm}^{-1}$ ) with a drift velocity depending on their size-to-charge ratio. A second RF ion funnel at the end of the drift tube collects and transports the now ion mobility-separated ions into the high vacuum region. After $\mathrm{m} / \mathrm{z}$ selection in a quadrupole mass filter, the timedependent ion current is recorded to obtain an arrival time distribution (ATD). Collision cross sections are determined using the MasonSchamp equation. ${ }^{42}$ Further details are given in the Supporting Information.

Infrared Spectroscopy. For ion mobility- and $m / z$-selective IR spectroscopy, a narrow ATD slice of $150 \mu$ s width is selected by electrostatic deflection prior to $\mathrm{m} / z$ selection. The ion mobility- and $\mathrm{m} / z$-selected ions are then irradiated by intense IR pulses provided by the Fritz Haber Institute free-electron laser (FHI FEL). ${ }^{43}$ When the IR wavelength is resonant with an infrared-active mode of the ion, the absorption of multiple photons can take place and dissociation can occur. The resulting fragment ions are monitored using a time-of-flight mass analyzer. IR spectra are obtained by scanning the laser in $3 \mathrm{~cm}^{-1}$ steps in the range $1000-1900 \mathrm{~cm}^{-1}$, averaging 20 mass spectra (laser shots) per step, and plotting the fragmentation efficiency as a function of wavenumber. Further details are given in the Supporting Information.

\section{RESULTS}

Ion Mobility Mass Spectrometry. A representative timeof-flight mass spectrum of PPIX and the ATDs that correspond to individual $\mathrm{m} / z$ selected ions are shown in Figure 2a,b. For many $m / z$ values, the corresponding ATDs contain several peaks. A priori, the masses and charges of the oligomeric species are unknown, as via the mass spectrum, only the $\mathrm{m} / z$ ratios are determined. However, in an ATD, the drift time of an ion is proportional to its $\mathrm{CCS} / z$ ratio. Assuming reasonable CCS values and taking the progression of the drift times as a function of $\mathrm{m} / z$ into account, the peaks in an ATD can be assigned to specific $n / z$ values. For the most intense signal in the mass spectrum at $m / z 563$, only one species is found in the ATD, which can be assigned to the singly protonated monomer $(n / z=1 / 1)$. Ions with $m / z 1127$ can be assigned to aggregates with $n / z=4 / 2$ and 2/1, while those with $\mathrm{m} / z 1691$ are likely to stem from the four separable species with $n / z=12 / 4,9 / 3,6 / 2$, and $3 / 1$. The $12 / 4$ aggregate is also the largest aggregate that is observable under the present experimental conditions. For comparison, we also sprayed PPIX from basic solution and recorded mass spectra (see Figure $S 1$ in the Supporting Information). Under those conditions, monomers and dimers are abundant, whereas trimers and tetramers are found only with low abundances and larger aggregates $(n>4)$ are not present. This is in line with previous reports, which suggest that no higher aggregates are formed in aqueous basic solution $(\mathrm{pH}$ $>$ 8). ${ }^{31,44}$

From the ATDs, the CCS of each aggregate can be obtained and is plotted in Figure $2 \mathrm{c}$ as a function of aggregation number $n$. The numerical values are given in Table S2 (Supporting Information). Interestingly, the CCS increases linearly with the aggregation number $n$, suggesting highly ordered single- stranded linear growth of the aggregates. ${ }^{45}$ A linear fit (CCS $=a n+b)$ of the CCS evolution yields a slope of $a=68 \AA^{2}$ and an intercept of $b=112 \AA^{2}$. This can be interpreted in terms of a shell area per monomer unit and a base area of the linearly growing strand, respectively, ${ }^{45}$ which tentatively suggests faceto-face stacking of the porphyrin units with a single-stranded growth of the PPIX aggregates (vide infra and Supporting Information). The role of the propionic acid tails in the aggregation, however, cannot be deduced from the ion mobility experiment, as the measured CCS values only provide information on the overall sizes and shapes of aggregates. Additional information can be obtained from IR spectroscopy.

IR Spectroscopy. The IRMPD spectra for aggregates in the size range from $n / z=1 / 1$ to $8 / 3$ are shown in Figure $3 a-f$.

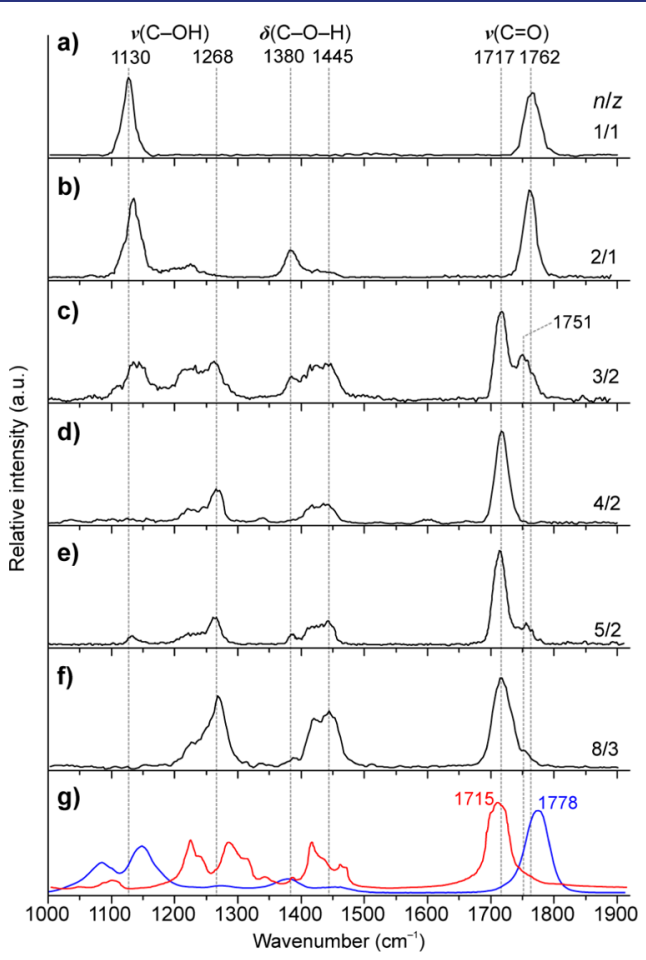

Figure 3. Ion mobility- and $m / z$-selected IRMPD spectra of protonated PPIX aggregates $[n \mathrm{PPIX}+z \mathrm{H}]^{z+}: n / z=$ (a) $1 / 1$, (b) $2 /$ 1 , (c) $3 / 2$, (d) $4 / 2$, (e) $5 / 2$, and (f) $8 / 3$. (g) Reference IR spectra of butanoic acid in the gas phase (blue) and in $\mathrm{CCl}_{4}$ (red), reproduced from NIST Chemistry WebBook. ${ }^{46}$

Representative photofragment mass spectra are shown in Figure S2 (Supporting Information). The spectrum of species $1 / 1$ is dominated by two bands at 1130 and $1762 \mathrm{~cm}^{-1}$. As the size increases, four additional bands at $1268,1380,1445$, and $1717 \mathrm{~cm}^{-1}$ begin to increase in intensity, while the former ones decrease in relative intensity.

In the mid-IR region, carboxylic acid groups have characteristic absorption bands for $\mathrm{C}=\mathrm{O}$ and $\mathrm{C}-\mathrm{OH}$ stretching vibrations as well as for the $\mathrm{C}-\mathrm{O}-\mathrm{H}$ bending mode. The positions of these bands strongly shift upon hydrogen bonding and can, thus, be highly diagnostic. For a band assignment, it is often useful to compare experimental results with IR spectra of simpler compounds of known structure that feature similar functional groups. In Figure 3g, IR spectra of neutral butanoic acid are shown for two different environments: ${ }^{46}$ the gas phase (blue), which favors the existence of individual monomers and therefore no hydrogen-bonding interactions of the carboxylic 


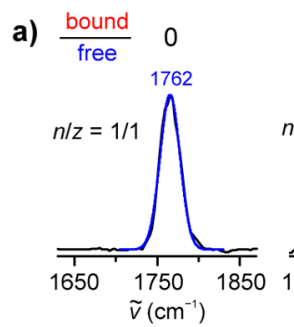

b)
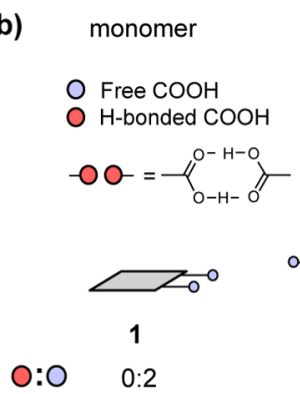

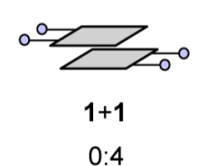

$0: 4$
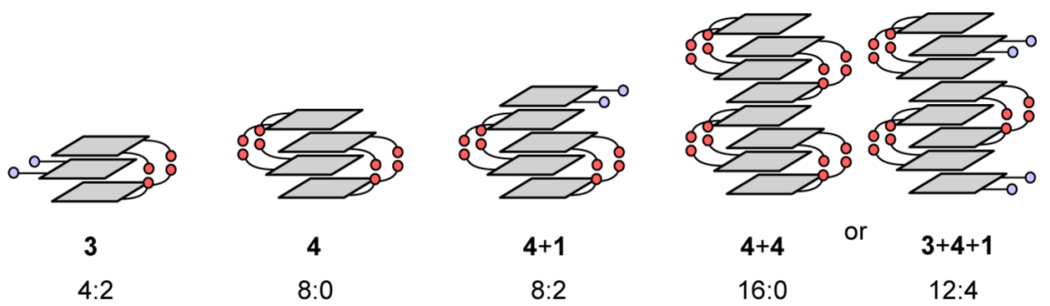

Figure 4. (a) IR spectra of PPIX aggregates in the wavenumber range for $\mathrm{C}=\mathrm{O}$ stretching vibration. The IR features are deconvoluted by two Gaussian profiles for hydrogen-bonded (red) and unbound (blue) $\mathrm{C}=\mathrm{O}$ stretching modes. The ratios between the hydrogen-bonded and the free carboxylic acids are determined from the areas of fitted Gaussians. (b) Proposed self-assembled geometries of PPIX aggregates. Blue and red circles denote free and hydrogen-bonded carboxylic acids, respectively.

acid groups, and for a nonpolar solvent (red) such as $\mathrm{CCl}_{4}$, where butanoic acid is known to form dimeric pairs held together by two $\mathrm{C}=\mathrm{O} \cdot \mathrm{HO}-\mathrm{C}$ hydrogen bonds (see also Figure 1c). These spectra can therefore serve as a reference for free and hydrogen-bonded carboxylic acids, respectively, and the IR bands can be assigned in terms of $\mathrm{C}=\mathrm{O}$ and $\mathrm{C}-\mathrm{OH}$ stretching $(\nu)$ and $\mathrm{C}-\mathrm{O}-\mathrm{H}$ bending $(\delta)$ vibrations as indicated by dotted vertical lines in Figure 3. Upon dimerization, the $\mathrm{C}=$ $\mathrm{O}$ stretch frequency exhibits a red shift of approximately 63 $\mathrm{cm}^{-1}$, while the $\mathrm{C}-\mathrm{OH}$ stretch frequency shifts toward blue by $\sim 140 \mathrm{~cm}^{-1}$, reflecting a decrease and increase in the carbonoxygen bond strength, respectively.

From visual comparison, it becomes clear that the spectra of $n / z=1 / 1$ and $2 / 1$ share many features with the spectrum of free, gas-phase butanoic acid monomers while those of the larger aggregates resemble more that of butanoic acid dimers in $\mathrm{CCl}_{4}$. Dimeric hydrogen bonding of propionic acid tails is not possible for $n / z=1 / 1$ and, from the spectra, does not seem to occur for $n / z=2 / 1$. For $n / z=3 / 2$, a red-shifted $\mathrm{C}=\mathrm{O}$ stretch band around $1717 \mathrm{~cm}^{-1}$ appears with significantly higher intensity compared to the original stretch band of free $\mathrm{C}=\mathrm{O}$. The IR spectrum of this aggregate resembles more that of the butanoic acid dimer. In the case of $n / z=4 / 2$, the band corresponding to a free $\mathrm{C}=\mathrm{O}$ stretch vibration is completely absent, however, comes back as a small band for $n / z=5 / 2$ and as a weak shoulder for $n / z=8 / 3$. These IR spectroscopic results suggest that the interaction of the propionic acid tails plays a role in aggregate formation.

\section{DISCUSSION}

Stacking Pattern. In order to estimate the relative abundance of bound and free propionic acids in the investigated PPIX aggregates, the IR bands resulting from the $\mathrm{C}=\mathrm{O}$ stretching mode between 1650 and $1800 \mathrm{~cm}^{-1}$ were fitted with a sum of two Gaussian profiles (see Figure 4a). The Gaussian profiles in red represent $\mathrm{C}=\mathrm{O}$ stretching vibrations for the paired propionic acids, whereas the blue curves stand for free propionic acids. The evolution of IR features and the determined bound/free ratios of propionic acids can then be used to deduce more detailed information on the geometries of PPIX assemblies. Together with results from the IM-MS experiments, which show that the PPIX aggregates grow as a linear stacking motif, a general growth pattern can be postulated (see Figure 4b).

Neither in the PPIX monomer nor in the dimer do the propionic acid tails interact with each other in the form of double $\mathrm{C}=\mathrm{O} \cdots \mathrm{HO}-\mathrm{C}$ hydrogen bonds. For the PPIX dimer, such an interaction is probably unfavorable due to steric constraints and, likely, the two hydrophilic tails are on different, possibly opposite sides when stacking occurs (see Figure 4b). In a previous study, a similar head-to-tail stacking geometry was suggested for a dimer at basic condition $(\mathrm{pH} \sim 12) .{ }^{31}$ Possible driving force for such alignment might be electrostatic (charge-charge) repulsions between tails that are deprotonated in a basic environment. However, even without any charge repulsion, the lack of interaction between tails indicates that steric constraints can influence their orientation.

A trimer can now be formed by stacking a third unit onto a dimer. For this aggregate, an interaction between the tails of the top and bottom unit seems sterically possible, allowing for two double hydrogen bond interactions between those four carboxylic acid groups, however, leaving the tails of the center unit without hydrogen-bonding partners. For this structure, the bound/free ratio of carboxylic acids should then be 4:2, which is in a good agreement with the experimental value of 2.1 deduced from the IR spectrum.

Upon addition of a fourth unit to this trimer, hydrogen bonding to the tails of the trimer-center unit is possible. Thus, for the tetramer, complete tail pairing can occur. Experimentally, this is confirmed by the absence of the IR band of a free $\mathrm{C}=\mathrm{O}$ group at $>1750 \mathrm{~cm}^{-1}$. Such a tight assembly with complete pairing of propionic acid tails should give this aggregate a high relative stability, which is also indicated by the high relative abundance of the tetramer in the ion mobility mass spectrum in Figure $2 b$. 
A pentamer can be formed by adding a monomer to a tetramer. In such an aggregate, the eight propionic acid tails of the tetramer remain hydrogen bonded while the propionic acid tails of the additional monomer should again give rise to IR intensity for the free $\mathrm{C}=\mathrm{O}$ stretching mode in a bound/free ratio of $8: 2$. Experimentally, a value of 4.6 is observed, in good agreement with the proposed model.

It should be noted that two assumptions go into the correlation of the bound/free ratios to the relative IR intensities. First, the IR absorption cross sections of free and bound $\mathrm{C}=\mathrm{O}$ groups need to be similar, which should be the case. Second and more important, it is assumed that the here observed IRMPD yield is linearly proportional to the IR absorption cross-section, which, due to the nonlinear nature of the excitation process, is often not strictly the case. ${ }^{32,47}$ However, for the weakly bound aggregates in the present work, only a few photons are required to induce dissociation, which should minimize nonlinear effects of the multiple photon process. This is supported by an observed near-linear IRMPD yield as a function of laser intensity (see Figure S4 in the Supporting Information). Therefore, a correlation of the here observed relative IR intensities to bound/free ratios is at least semiquantitatively valid.

The stacking of higher aggregates can be described in terms of building up from smaller units, and the monomer (1), trimer (3), and tetramer (4) can serve as basic building blocks. In such a model, the stacked geometry of two tetramers $(4+4)$ is particular interesting, as it is the next unit which is not expected to possess any free propionic acid groups. Experimentally, for $n / z=8 / 3$, a small IR feature at $\sim 1751 \mathrm{~cm}^{-1}$ indicates the presence of free propionic acid. Interestingly, the photofragment mass spectra of this species show different fragmentation patterns, depending on whether excitation occurs on the band of hydrogen bonded $\mathrm{C}=\mathrm{O}$ at $1717 \mathrm{~cm}^{-1}$ or on the band of free $\mathrm{C}=\mathrm{O}$ at $1750 \mathrm{~cm}^{-1}$ (see Figure $\mathrm{S} 2$ in the Supporting Information). At $1717 \mathrm{~cm}^{-1}$, the most intense fragment ion is the doubly charged tetramer $(4 / 2)$ ion. At $1750 \mathrm{~cm}^{-1}$, on the other hand, the most intense fragment is the $5 / 2$ aggregate. These observations can be explained by the presence of two isomers (see Figure 4b) where cleavage preferentially occurs at positions, where no tail hydrogen bonds need to be broken. The first isomer can thus consist of a $(4+4)$ structure, which splits into two tetramers after excitation. The second possibly consists of a $(3+4+1)$ structure, which has four free propionic acid tails that absorb light at the respective band position. Upon excitation, it loses with high probability a trimer.

From the observed linear evolution of the CCS values, it can be deduced that the PPIX aggregates are likely to have a singlestrand face-to-face stacking geometry. The spectroscopic results indicate a pairing of the propionic acid tails from unit $n$ to unit $n+2$. The length of a dimeric pair of the propionic acid tails in PPIX is about 7-8 $\AA$. The interplanar distance in the face-toface stacking of porphyrins is typically $\sim 3.4 \AA{ }_{;}^{29,48}$ the distance between an $n$ and $n+2$ PPIX unit thus fits to the length of the dimeric pairs of propionic acids. This emphasizes that steric and geometric factors, in this case the length of propionic acid tails, are critical to stabilize the assembly.

To assess the viability of this stacking model, we performed molecular modeling for $n / z=$ from $1 / 1$ to $8 / 3$. Details are shown in Figure S5 (Supporting Information). The geometries obtained are in agreement with the qualitative model described above. The plane-to-plane distance is 3.5-4.0 $\AA$, which is a typical interplanar distance of $\pi$-stacked porphyrins. The dimeric pairing of propionic acid tails is stable and not hindered by steric constraints. The calculated slip angles between planes are $\sim 50^{\circ}$, which is in line with previous observations of the slip angle in PPIX aggregates. ${ }^{31}$ Theoretical CCS values are slightly smaller than the experimental values, but still in good agreement. Importantly, the calculated CCS increases linearly with the aggregation number, which supports the single-strand growth of PPIX with consecutive face-to-face stacking.

Relevance to Condensed-Phase Properties. In how far do the here presented results reflect the situation in solution? The experimental setup has been designed to allow for a gentle transition of the ions into the gas phase and through the instrument. The concentration of the solute in the here presented experiments is rather low. In addition, the nanoESI tip is carefully prepared to make the tip opening as small as possible and the electrospray voltage is kept very low (0.6-0.7 $\mathrm{kV}$ ) in order to minimize nonspecific aggregation by reducing the initial size of the electrospray droplets. ${ }^{49}$ When recording mass spectra, the trimer $(n / z=3 / 2)$ and tetramer $(n / z=4 / 2)$ are pronounced even at low sample concentration $(10 \mu \mathrm{M})$, while at the same time the dimer $(n / z=2 / 1)$ is barely observable (see Figure S6 in the Supporting Information). This suggests that at least these aggregates with paired propionic acid tails are not nonspecific aggregates that form during the electrospray process, but specific aggregates that are already present in solution. We can thus expect that the here observed distribution of aggregates as well as their structures represent to a large degree the distributions and structures that are present in solution.

The experiments are performed on protonated species and the presence of a charge can influence the structures of the aggregates as well as the position of IR bands. Under the experimental conditions, the propionic acid tails will not carry any charges, since the $\mathrm{p} K_{\mathrm{a}}$ value of propionic acid is $\sim 9.7$ in methanol, ${ }^{51}$ ruling out their deprotonation. The locations of the extra protons will be at the most basic sites of the aggregates, which are the nitrogen atoms of the porphine ring. The normally flat porphine ring is known to slightly distort upon protonation. However, studies on the non-covalent interactions between protonated porphyrin and fullerenes indicate that $\pi-\pi$ interactions are still maintained. ${ }^{4}$ Therefore, even when protonated in the gas phase, the main structural features of the PPIX aggregates in solution may be still retained.

Comparison with Other Models. For porphyrin derivatives, the hydrophilic substituents often provide stabilization via hydrogen bonding to form highly extended stacking structures. For example, the PPIX derivatives with 2-aminoglycosamide head groups form long fibrils, ${ }^{6,7}$ and other amide-derived PPIXs containing triethylene glycol (TEG) form spherical vesicles or micelles depending on the solvent composition. ${ }^{30}$ Similarly, meso-substituted porphyrins containing alkylamide groups form nanowires. ${ }^{8,9}$ In those systems, the amide groups are suggested to form cooperative hydrogen-bonding networks along the stacking axis, which greatly stabilize the elongated face-to-face stacked structure. In addition, the bulky or long chain groups after the amide bond can interact with solvents to gain additional stabilization energy. ${ }^{10-13,30}$

It appears that in PPIX, propionic acid tail pairing between the $n$ and $n+2$ units makes the tetrameric assembly highly stable and gives rise to a pronounced $4 / 2$ peak in the mass spectrum. In such an aggregate, there is no free propionic acid tail available to bind additional units. This might be one reason 
why PPIX has unique characteristics and does not form highly ordered fibril-like structures but rather globular aggregates as additional units are only bound by relatively weak stacking interactions.

In the amide-derived PPIX aggregates, the amide $\mathrm{C}=\mathrm{O}$ and $\mathrm{N}-\mathrm{H}$ groups are well aligned along the stacking axis, and both of them can form H-bonds independently with $n-1$ and $n+1$ units, resulting in a continuous chain of hydrogen bonds. On the other hand, the hydrogen-bonding properties of carboxylic acid groups are different, as they prefer strong one-to-one hydrogen binding with only one other group (see Figure 1c), resulting in a $n / n+2$ interaction. These intrinsic properties of propionic acid tails, together with the presence of stable tetramers, thus hinder the formation of extended hydrogenbonding networks.

Alternatively, two propionic acid tails may be aligned in opposite directions, with one binding to a $n+2$ and the other to the $n-2$ unit (Figure $5 \mathrm{~b}$ ), which would allow the formation of a)

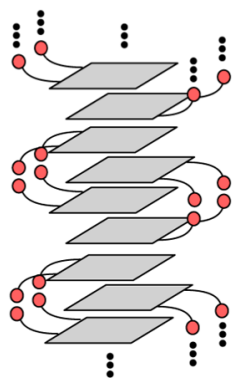

b)

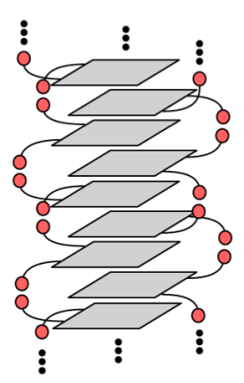

Figure 5. Two possible arrangements of paired propionic acid tails. Two tails in a PPIX can be oriented (a) in same directions and (b) in opposite directions.

stable, extended structures connected by consecutive hydrogen bonds of propionic acid tails. However, for smaller aggregates and during growth, such an arrangement is energetically not favorable, and for larger aggregates, the formation of such a structure would then require the rearrangement of a large portion of the hydrogen-bonding network such that those structures do not form.

Previously, a similar arrangement as shown in Figure 5 b was suggested for partly deprotonated PPIX assemblies. ${ }^{31}$ Since deprotonated carboxylic acid can exist in aqueous solution at neutral $\mathrm{pH}$, intermolecular $\mathrm{COOH} \cdots{ }^{-} \mathrm{OOC}$ interaction is suggested to be the main interactions between tails. Due to charge repulsion, the here suggested structures (Figure 5a), might not be stable with deprotonated tails. However, IM-MS results (see Figure S1 in the Supporting Information) clearly confirm the formation of the tetramer (4) and trimer (3) even under basic conditions. Their formation can impede the growth of extended structures like the ones shown Figure $5 \mathrm{~b}$ and cause the previously observed instability of large assemblies in aqueous solution. ${ }^{31}$

How can we use the present findings to design and build highly ordered nanostructures? PPIX as such is clearly not a suitable building block for extended supramolecular assemblies. This may be a favorable property of this molecule, since PPIX is commonly occurring in biological systems. For PPIX, the main hindrance for forming extended assemblies is the formation of a stable tetramer with no free hydrogen-bonding partners in its tails. When designing molecules suitable for 1-D supramolecular structures such a possibility should be avoided.
Amide based PPIX derivatives, on the other hand, appear more suitable for forming extended structures, as the tail can bind simultaneously to the layer below as well as above. In such an arrangement, the ends of a 1-D structure always provide a hydrogen-bonding partner for docking a further unit.

\section{CONCLUSIONS}

This work shows the capability of the combined IM-MS and gas-phase IR spectroscopic approach to investigate supramolecular assemblies, especially to identify the assembly behavior and mechanisms of early aggregates, which are otherwise difficult to deduce using traditional condensedphase methods.

Here, details of the early self-assembly of PPIX are deduced from the IR spectra as well as from the CCS values of individual isolated aggregates. The evolution of the measured CCS as a function of aggregate size indicates a single-stranded face-toface stacking. The evolution of IR features suggests the pairing of propionic acid tails between the $n$ and $n+2$ units. This alternating pairing yields a tight tetrameric assembly that might inhibit the formation of large elongated fibril-like PPIX structures. In the future, this information on the early PPIX aggregates might help in the design and tailoring of various supramolecular structures that are based on PPIX derivatives.

\section{ASSOCIATED CONTENT}

\section{Supporting Information}

The Supporting Information is available free of charge on the ACS Publications website at DOI: 10.1021/jacs.6b08700.

Further experimental details, mass spectra of PPIX in basic solution, collision cross sections and photofragmentation mass spectra of protonated PPIX monomers and aggregates, possible dissociation pathways of PPIX aggregates, effect of laser pulse energy on IR intensity, molecular modeling results, and effect of PPIX concentration on the aggregate distribution (PDF)

\section{AUTHOR INFORMATION}

\section{Corresponding Author}

*helden@fhi-berlin.mpg.de

ORCID ${ }^{\text {ㅇ }}$

Jongcheol Seo: 0000-0001-5844-4585

Wieland Schöllkopf: 0000-0003-0564-203X

Gert von Helden: 0000-0001-7611-8740

Notes

The authors declare no competing financial interest.

\section{ACKNOWLEDGMENTS}

J.J. acknowledges the support of the 2014 Global Internship Program from Korean Council for University Education.

\section{REFERENCES}

(1) Olmstead, M. M.; Costa, D. A.; Maitra, K.; Noll, B. C.; Phillips, S. L.; Van Calcar, P. M.; Balch, A. L. J. Am. Chem. Soc. 1999, 121, 70907097.

(2) Boyd, P. D. W.; Reed, C. A. Acc. Chem. Res. 2005, 38, 235-242.

(3) Shirakawa, M.; Fujita, N.; Shinkai, S. J. Am. Chem. Soc. 2003, 125, 9902-9903.

(4) Jung, S.; Seo, J.; Shin, S. K. J. Phys. Chem. A 2010, 114, 1137611385 .

(5) Jung, S.; van Paauwe, J. D.; Boyd, P. D. W.; Shin, S. K. Phys. Chem. Chem. Phys. 2011, 13, 20248-20254. 
(6) Fuhrhop, J. H.; Demoulin, C.; Boettcher, C.; Koening, J.; Siggel, U. J. Am. Chem. Soc. 1992, 114, 4159-4165.

(7) Fuhrhop, J. H.; Bindig, U.; Siggel, U. J. Am. Chem. Soc. 1993, 115, 11036-11037.

(8) Shirakawa, M.; Fujita, N.; Shinkai, S. J. Am. Chem. Soc. 2005, 127, 4164-4165.

(9) Kishida, T.; Fujita, N.; Sada, K.; Shinkai, S. J. Am. Chem. Soc. 2005, 127, 7298-7299.

(10) Bhosale, S. V.; Bhosale, S. V.; Shitre, G. V.; Bobe, S. R.; Gupta, A. Eur. J. Org. Chem. 2013, 2013, 3939-3954.

(11) Bhosale, S. V.; Kalyankar, M. B.; Bhosale, S. V.; Patil, S. G.; Lalander, C. H.; Langford, S. J.; Nalage, S. V. Supramol. Chem. 2011, 23, 263-268.

(12) Bhosale, S. V.; Kalyankar, M. B.; Nalage, S. V.; Bhosale, S. V.; Lalander, C. H.; Langford, S. J. Supramol. Chem. 2011, 23, 563-569.

(13) Bhosale, S. V.; Nalage, S. V.; Booth, J. M.; Gupta, A.; Bhargava,

S. K.; Bhosale, S. V. Supramol. Chem. 2012, 24, 779-786.

(14) Segade, A.; Castella, M.; López-Calahorra, F.; Velasco, D. Chem. Mater. 2005, 17, 5366-5374.

(15) Engelkamp, H.; Middelbeek, S.; Nolte, R. J. M. Science 1999, 284, 785-788.

(16) Castella, M.; Lopez-Calahorra, F.; Velasco, D.; Finkelmann, H. Chem. Commun. 2002, 2348-2349.

(17) Kimura, M.; Wada, K.; Ohta, K.; Hanabusa, K.; Shirai, H.; Kobayashi, N. J. Am. Chem. Soc. 2001, 123, 2438-2439.

(18) Patel, B. R.; Suslick, K. S. J. Am. Chem. Soc. 1998, 120, 1180211803

(19) Gao, W.-Y.; Chrzanowski, M.; Ma, S. Chem. Soc. Rev. 2014, 43, 5841-5866.

(20) Beletskaya, I.; Tyurin, V. S.; Tsivadze, A. Y.; Guilard, R.; Stern, C. Chem. Rev. 2009, 109, 1659-1713.

(21) Schwarz, U.; Vonderach, M.; Armbruster, M. K.; Fink, K.; Kappes, M. M.; Weis, P. J. Phys. Chem. A 2014, 118, 369-379.

(22) Morisue, M.; Hoshino, Y.; Shimizu, K.; Shimizu, M.; Kuroda, Y. Chem. Sci. 2015, 6, 6199-6206.

(23) Shirakawa, M.; Kawano, S.-i.; Fujita, N.; Sada, K.; Shinkai, S. J. Org. Chem. 2003, 68, 5037-5044.

(24) Cai, J.; Chen, H.; Huang, J.; Wang, J.; Tian, D.; Dong, H.; Jiang, L. Soft Matter 2014, 10, 2612-2618.

(25) Wang, J.; Zhong, Y.; Wang, L.; Zhang, N.; Cao, R.; Bian, K.; Alarid, L.; Haddad, R. E.; Bai, F.; Fan, H. Nano Lett. 2016, 16, 65236528.

(26) Zhong, Y.; Wang, J.; Zhang, R.; Wei, W.; Wang, H.; Lü, X.; Bai, F.; Wu, H.; Haddad, R.; Fan, H. Nano Lett. 2014, 14, 7175-7179.

(27) Imahori, H.; Fukuzumi, S. Adv. Funct. Mater. 2004, 14, 525536.

(28) Yamada, H.; Imahori, H.; Nishimura, Y.; Yamazaki, I.; Ahn, T. K.; Kim, S. K.; Kim, D.; Fukuzumi, S. J. Am. Chem. Soc. 2003, 125, 9129-9139.

(29) Hunter, C. A.; Sanders, J. K. M. J. Am. Chem. Soc. 1990, 112, $5525-5534$.

(30) Bhosale, S. V.; Bhosale, S. V.; Kalyankar, M. B.; Langford, S. J.; Lalander, C. H. Aust. J. Chem. 2010, 63, 1326-1329.

(31) Scolaro, L. M.; Castriciano, M.; Romeo, A.; Patanè, S.; Cefalì, E.; Allegrini, M. J. Phys. Chem. B 2002, 106, 2453-2459.

(32) Oomens, J.; Sartakov, B. G.; Meijer, G.; von Helden, G. Int. J. Mass Spectrom. 2006, 254, 1-19.

(33) Seo, J.; Warnke, S.; Gewinner, S.; Schöllkopf, W.; Bowers, M. T.; Pagel, K.; von Helden, G. Phys. Chem. Chem. Phys. 2016, 18, 25474-25482.

(34) Warnke, S.; Seo, J.; Boschmans, J.; Sobott, F.; Scrivens, J. H.; Bleiholder, C.; Bowers, M. T.; Gewinner, S.; Schöllkopf, W.; Pagel, K.; von Helden, G. J. Am. Chem. Soc. 2015, 137, 4236-4242.

(35) Masson, A.; Kamrath, M. Z.; Perez, M. A. S.; Glover, M. S.; Rothlisberger, U.; Clemmer, D. E.; Rizzo, T. R. J. Am. Soc. Mass Spectrom. 2015, 26, 1444-1454.

(36) Voronina, L.; Masson, A.; Kamrath, M.; Schubert, F.; Clemmer, D.; Baldauf, C.; Rizzo, T. J. Am. Chem. Soc. 2016, 138, 9224-9233.
(37) Seo, J.; Hoffmann, W.; Warnke, S.; Huang, X.; Gewinner, S.; Schöllkopf, W.; Bowers, M. T.; von Helden, G.; Pagel, K. Nat. Chem. 2016, DOI: $10.1038 /$ nchem.2615.

(38) Seo, J.; Hoffmann, W.; Warnke, S.; Bowers, M. T.; Pagel, K.; von Helden, G. Angew. Chem., Int. Ed. 2016, 55, 14173-14176.

(39) Warnke, S.; von Helden, G.; Pagel, K. Proteomics 2015, 15, 2804-2812.

(40) Kemper, P. R.; Dupuis, N. F.; Bowers, M. T. Int. J. Mass Spectrom. 2009, 287, 46-57.

(41) Warnke, S.; Baldauf, C.; Bowers, M. T.; Pagel, K.; von Helden, G. J. Am. Chem. Soc. 2014, 136, 10308-10314.

(42) Mason, E. A.; McDaniel, E. W. Transport Properties of Ions in Gases; Wiley-VCH: New York, 2005.

(43) Schöllkopf, W.; Gewinner, S.; Junkes, H.; Paarmann, A.; von Helden, G.; Bluem, H.; Todd, A. M. M. Proc. SPIE 2015, 9512, 95121L.

(44) Inamura, I.; Uchida, K. Bull. Chem. Soc. Jpn. 1991, 64, 20052007.

(45) Bleiholder, C.; Dupuis, N. F.; Wyttenbach, T.; Bowers, M. T. Nat. Chem. 2011, 3, 172-177.

(46) Stein, S. E. In NIST Chemistry WebBook, NIST Standard Reference Database Number 69; Linstrom, P. J., Mallard, W. G., Eds.; National Institue of Standards and Technology: Gaithersburg, MD, 2015.

(47) Prell, J. S.; Chang, T. M.; O’Brien, J. T.; Williams, E. R. J. Am. Chem. Soc. 2010, 132, 7811-7819.

(48) Mück-Lichtenfeld, C.; Grimme, S. Mol. Phys. 2007, 105, 27932798.

(49) Davidson, K. L.; Oberreit, D. R.; Hogan, C. J.; Bush, M. F. Int. J. Mass Spectrom. 2016, DOI: 10.1016/j.ijms.2016.09.013.

(50) Lane, L. A.; Ruotolo, B. T.; Robinson, C. V.; Favrin, G.; Benesch, J. L. P. Int. J. Mass Spectrom. 2009, 283, 169-177.

(51) Rived, F.; Rosés, M.; Bosch, E. Anal. Chim. Acta 1998, 374, 309-324. 